\title{
A Study on Cortico-muscular Coupling in Finger Motions for Exoskeleton Assisted Neuro-Rehabilitation
}

Chowdhury, A., Raza, H., Dutta, A., Nishad, S. S., Saxena, A., \& Prasad, G. (2015). A Study on Corticomuscular Coupling in Finger Motions for Exoskeleton Assisted Neuro-Rehabilitation. In Unknown Host Publication (pp. 4610 -4614). IEEE. https://doi.org/10.1109/EMBC.2015.7319421

Link to publication record in Ulster University Research Portal

\author{
Published in: \\ Unknown Host Publication
}

Publication Status:

Published (in print/issue): 25/08/2015

DOI:

10.1109/EMBC.2015.7319421

\section{Document Version}

Author Accepted version

\section{General rights}

Copyright for the publications made accessible via Ulster University's Research Portal is retained by the author(s) and / or other copyright owners and it is a condition of accessing these publications that users recognise and abide by the legal requirements associated with these rights.

\section{Take down policy}

The Research Portal is Ulster University's institutional repository that provides access to Ulster's research outputs. Every effort has been made to ensure that content in the Research Portal does not infringe any person's rights, or applicable UK laws. If you discover content in the Research Portal that you believe breaches copyright or violates any law, please contact pure-support@ulster.ac.uk. 


\title{
A Study on Cortico-muscular Coupling in Finger Motions for Exoskeleton Assisted Neuro-Rehabilitation
}

\author{
Anirban Chwodhury ${ }^{1}$, Haider Raza ${ }^{2}$, Ashish Dutta ${ }^{1}$, Shyam Sunder Nishad $^{1}$, Anupam Saxena ${ }^{1}$, \\ Girijesh Prasad ${ }^{2}$ \\ ${ }^{1}$ IIT Kanpur, Kanpur, India; ${ }^{2}$ ISRC, Ulster University, Magee Campus, Derry Londonderry, N. Ireland, UK \\ anir@iitk.ac.in, raza-h@email.ulster.ac.uk, adutta@iitk.ac.in, shyam@iitk.ac.in, anupams@iitk.ac.in, \\ g.prasad@ulster.ac.uk
}

\begin{abstract}
In this paper our objective is to analyze the cortico-muscular coupling for hand finger motion and its possible use in the control of an exoskeleton based neurorehabilitation system for stroke sufferers. Cortical activity alone is often not sufficient to reliably control a device such as an exoskeleton and hence, our focus is to ascertain and analyze the connectivity between the motor cortex and forearm muscles, controlling the fingers, in terms of coherence between electroencephalogram (EEG) and electromyogram (EMG) signals. We have analyzed the signals separately for three different kinds of exercises consisting of passive motion of fingers using exoskeleton, active motion without any assistance, and motor imagery of the same movements. Four out of six healthy subjects who participated in the experiments have shown significant $(p<0.01)$ coherence for active finger motion which is well distinguished from the rest state. The EEG analysis resulted in average accuracy of $69.17 \%$ for passive finger motion with exoskeleton, $71.25 \%$ for active finger motion, and $67.92 \%$ for motor imagery, in detecting the volitional intention of the subjects to move their fingers. These results support that EEG-EMG coherence along with EEG analysis has the potential to make a more effective neurorehabilitation system for finger movement restoration of stroke sufferers.
\end{abstract}

Keywords-Coherence, EEG, EMG, Exoskeleton, Rehabilitation.

\section{INTRODUCTION}

The total number of people suffering from stroke annually is over 20 million, and almost $40 \%$ of them suffer from upper-limb paralysis, which drastically affects their normal lifestyle and employability [1]. Effective motor recovery after stroke requires intensive voluntary practice of activities of daily living (ADL), using their paretic limb [2]. These exercises reorganize the neural networks in the affected areas of the brain, which is called the neuroplasticity [3].

There are many devices for assisting stroke patients for neuro-rehabilitation. The EMG based hand rehabilitation systems have been developed by extracting EMG signals from the Abductor Pollicis-Brevis, and Extensor-Digitorum muscles to trigger an Exoskeleton with $20 \%$ of maximum volumetric contraction (MVC)[4]. Researchers are also able to relate the finger positions and the force generation by the finger tips using EMG signals [5].Although these studies have successfully been implemented for different hand rehabilitation systems, they suffer from a common shortcoming, which is, how much these EMG signals are due to the volitional intension of the persons. This is because in case of muscle spasticity EMG signals may not be coordinated with the brain activity and can be both voluntary and involuntary [6]. There are also several studies, which use brain signals only to give a neuro-feedback to the patients [7]. EEG signals have been proposed to be used to capture patients kinesthetic imagination for controlling the virtual gaming consoles [7]. EEG-based BCI systems are also built for giving neuro-feedback by means of moving a virtual hand on the screen while the participant is doing motor imagery [8]. Although some of these systems have shown slight improvement in neuro-plasticity after the end of certain number of training sessions, they do not focus on how much these brain-activities are influencing the EMG signal generation in motor-units in the muscles. Several researchers have shown that synchronized cortical rhythms are deeply connected with the generation of motor unit activities [9]. This synchronization can also be used to trigger external devices [10]. However, different subjects could show different levels of synchronization, which could also vary in the frequency ranges where they are appearing, as the capability of neurons to work in synchrony varies[11]. Also the amount of conscious control over a muscular activity determines the quality of synchronization that could be achieved [12]. In recent studies, synchronicity has been found for large movements such as human walking or in static contraction of Tibialis anterior muscle for at least 30\% of MVC [13]. In another study, the cortico-muscular connectivity for wrist movements on the affected side of the brain of the stroke patients have been investigated [14]. A method to estimate the latency of conduction time from EEG to EMG has also been proposed [15].A multivariate autoregressive modeling was used for plausible EEG-EMG connection, which is a time domain approach [16]. In the case of subtle finger movements, which require lesser conscious effort, it is difficult to obtain the synchronicity, and also the muscle fatigue may decrease the cortico-muscular coupling [17]. Besides there are few more challenges in detecting the cortical activity for finger motions, such as low spatio-temporal resolution, lower SNR, and the involvement of lower muscle mass [18].

Here we aim to investigate the possibility of using the cortico-muscular coupling between the EEG and EMG from 
the forearm muscles for activating an exoskeleton based rehabilitation device for people who suffer from the loss of mobility of their hand fingers. This brain to muscle coordination is hard to find in acute stages of disability after stroke. Therefore the system must also be supplemented with EEG activity analysis to detect volitional intention detection, as the severity of the disability demands. In this study, we have focused on how to distinguish a finger motion activity from the finger rest period for three different exercises consisting of passive movement by wearing a three finger exoskeleton, active finger movement without any exoskeleton, and the kinesthetic imagination of the same movement, by using the EEG signals. Further we have also investigated the coupling between the cortical activity and the muscular activity for each individual finger, i.e. Index finger (IF), Middle finger (MF), and Thumb (TH). We have used the standard technique of calculating the cortico-muscular coupling by using coherence between the frequency spectrum of the EEG and EMG signals, which is given as follows:

$$
C_{x y}(\lambda)=\frac{\left|f_{x y}(\lambda)\right|^{2}}{f_{x x}(\lambda) f_{y y}(\lambda)}
$$

where, $f_{x y}(\lambda)$ is Cross-Spectral Density of signals $\mathrm{x}$ and y. $f_{x x}(\lambda), f_{y y}(\lambda)$ are Auto-Spectral Density of $\mathrm{x}$ and $\mathrm{y}$ accordingly [19].

The basic architecture of the proposed rehabilitation system is shown in Fig.1

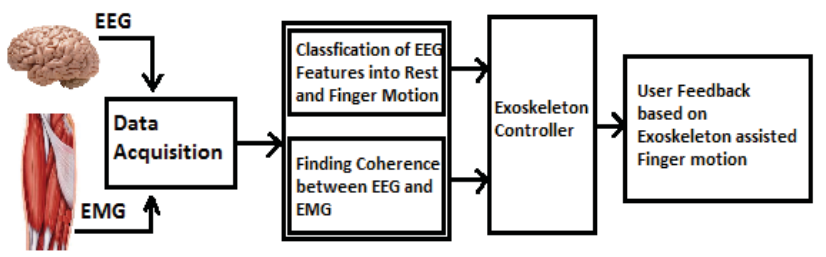

Fig.1. Basic architecture of the proposed rehabilitation system

This paper proceeds as follows: Section II presents an experimental paradigm. Section III shows the signal acquisition and analysis. Section IV presents the result. Finally, the discussion is given in Section V.

\section{EXPERIMENTAL PARADIGM}

Experiments were conducted on 6 consenting healthy subjects [S1-S6] aged between 20 and 30 years. The subjects were asked to undergo 3 different kinds of exercises. The first exercise was repetitive flexion and extension of fingers, while wearing a hand exoskeleton that is mounted only on 3 fingers (i.e., IF, MF, and TH). The second exercise was the same, except the subjects had to exercise actively without wearing the exoskeleton and the third exercise was the motor imagery (MI) of the same task without overt movement of the fingers. Each type of exercise was conducted in 3 runs by giving 4 minutes break in between. Each run consisted of 40 trials of 11 second duration. This time period is divided in the following way. First 3 seconds is the fixation cross. After that a cue appears on the screen to instruct the subject whether the exercise is to be performed or not. Then accordingly the subjects need to respond for that action for the next 8 seconds. A rest period between 1 and 2 second is given before going into the next trial. The timing diagram of a trial is shown in Fig.2. During the entire process of experimentation both the EEG and EMG signals were recorded simultaneously, and stored for further analysis. The EEG signals were recorded over the motor cortex from 10 channels. Among them $\mathrm{CP} 1, \mathrm{CP} 3, \mathrm{CPz}, \mathrm{CP} 4, \mathrm{CP} 2$ were connected in bipolar mode with respect to $\mathrm{FC} 1, \mathrm{FC} 3, \mathrm{FCz}$, $\mathrm{FC} 4, \mathrm{FC} 2$, respectively, i.e. $\mathrm{C} 1, \mathrm{C} 3, \mathrm{Cz}, \mathrm{C} 4$, and $\mathrm{C} 2$ were recorded in bipolar mode. The ground electrode was placed on the forehead and the reference electrode was attached to one of ear lobes.

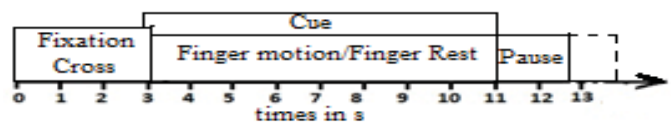

Fig.2. Timing Diagram of a single trial

The EMG signals were recorded from the lower arm using 3 bipolar channels, among which two were placed on the Flexor Digitorum-Superficialis muscle, and one was on the Flexor-Policus-Longus muscle. The reference was placed on the bony part of the elbow. The EEG signals were band passed between $0.1 \mathrm{~Hz}$ and $100 \mathrm{~Hz}$, and the EMG signals between $5 \mathrm{~Hz}$ and $100 \mathrm{~Hz}$, with a notch filter at $50 \mathrm{~Hz}$ for rejecting the noise due to $\mathrm{AC}$ power source, in both the cases. The sampling frequency was set to be $512 \mathrm{~Hz}$ for both the EEG and EMG signals. The electrode placements and the developed exoskeleton are shown in Fig.3. There are three degrees-of-freedom for each finger in the Exoskeleton which are designed by four bar linkages and are driven by servo motors [20].

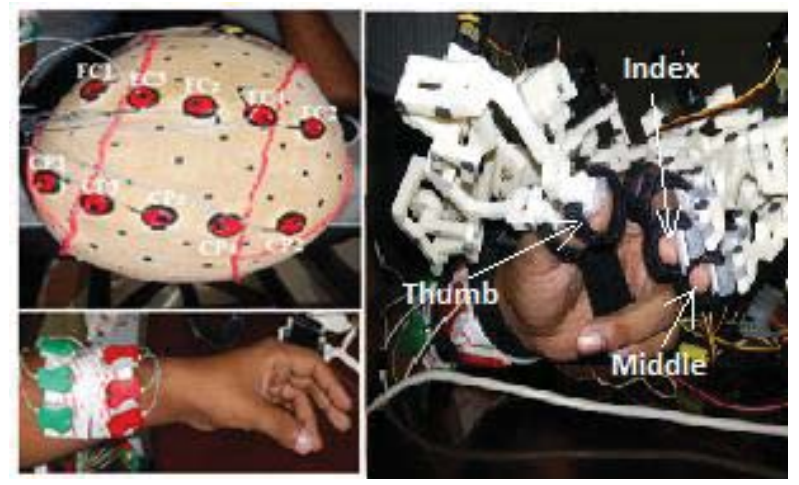

Fig. 3 The EEG and EMG electrode placements(Left) and The Three Finger Exoskeleton mounted on subject's hand(Right).

\section{ANALYSIS OF THE SIGNALS}

Based on the paradigm timing information, the time series data over a trial period was considered as one epoch and the epochs were arranged in a two dimensional matrix. Each column contained a new epoch having $5632(512 \mathrm{~Hz}$ x $11 \mathrm{~s}$ $=5632$ ) elements. The epochs, wherein the cue was given for execution, are categorized as class 1 , and for non-execution as class 2. The objective of the analysis was to train a classifier, which distinguishes these two classes by looking at some features of the EEG signals contained within a particular epoch and also to find out the coupling between the EEG and EMG signals in terms of coherence. The band power features were extracted out of the EEG signals in the range $\mathrm{mu}$ band $(8-12 \mathrm{~Hz})$ and beta band $(16-24 \mathrm{~Hz})$. These band power features are calculated for the entire $3 \mathrm{~s}$ to $11 \mathrm{~s}$ 
period with $1 \mathrm{~s}$ of sliding window length and $0.75 \mathrm{~s}$ of overlap, i.e. a resolution of $0.25 \mathrm{~s}$ is taken. The mean of 3 seconds period before the appearance of the cue is taken as the baseline. The LDA classifier is trained by taking the features of each of these time instants at a time from all the trials to find out at which time instant the classification accuracy is maximum. The 3 runs of 40 trials are combined together (i.e., 120 trials), among which 80 trials were kept for training the LDA classifier, and the rest 40 trials were kept for evaluation. A 5-fold cross-validation (5-CV) is performed on the training set. Next, the evaluation is performed on the test data, which was not included in the training set. The classifiers are built separately for the three different exercises, and their accuracies are compared. To find out the coupling between the EEG and EMG signals, the band pass filtered data from a particular trial, is fed into a MATLAB function called 'mscohere' to get the distribution of Coherence Coefficient across the frequency range 1-40 Hz. The travelling window and overlap parameters of this function were set to default (i.e., window size sufficient enough to divide the data-length into eight equal sections and overlap is the half of the window length) and the frequency resolution was of $1 \mathrm{~Hz}$, i.e. the n-point DFT points were taken same as the sampling frequency. Then the coherence distributions are averaged over all 120 trials to get an overall estimate.

\section{RESULTS}

The classification accuracies in percentage $(\%)$ calculated for each subject for the three different exercises are shown in Table.1 -Table.3. And the Coherence patterns are shown in Fig.4. The Table.1 shows the result of passive movement (assisted by the exoskeleton). Table.2 presents the result of the active movement (movement of the fingers without any assistance, and Table. 3 presents the motor imagery results.

TABle. 1 Classification ACCURACIES In CASE OF PASSIVE EXECUTION

\begin{tabular}{|c|c|c|c|c|c|}
\hline Sub & Band & $\begin{array}{c}\text { Time } \\
\text { (s) }\end{array}$ & Channel & $\begin{array}{c}\text { 5-CV } \\
(\%)\end{array}$ & $\begin{array}{l}\text { Eval } \\
(\%)\end{array}$ \\
\hline S1 & $\mu$ and $\beta$ & 5 & $\mathrm{C} 1, \mathrm{C} 3, \mathrm{C} 2$ & 76.25 & 72.5 \\
\hline S2 & $\mu$ and $\beta$ & 4.7 & $\mathrm{C} 1, \mathrm{C} 3, \mathrm{Cz}$ & 66.25 & 67.5 \\
\hline S3 & $\mu$ and $\beta$ & 3.5 & $\mathrm{C} 1, \mathrm{C} 3, \mathrm{C} 2$ & 72.5 & 75 \\
\hline S4 & $\mu$ only & 5.5 & $\mathrm{C} 3, \mathrm{Cz}, \mathrm{C} 4, \mathrm{C} 2$ & 62.5 & 65 \\
\hline S5 & $\mu$ and $\beta$ & 3.25 & $\mathrm{C} 1, \mathrm{Cz}, \mathrm{C} 4$ & 68.75 & 70 \\
\hline S6 & $\mu$ and $\beta$ & 6.75 & $\mathrm{Cl}, \mathrm{Cz}$ & 66.25 & 65 \\
\hline \multicolumn{4}{|c|}{ Mean } & 68.75 & 69.17 \\
\hline
\end{tabular}

TABle. 2 Classification ACCURACIES In CASE OF HAND EXECUTION

\begin{tabular}{|l|l|l|l|l|l|}
\hline Sub & Band & $\begin{array}{c}\text { Time } \\
(\mathbf{s})\end{array}$ & \multicolumn{1}{|c|}{ Channel } & $\begin{array}{c}\text { 5-CV } \\
(\mathbf{\%})\end{array}$ & $\begin{array}{c}\text { Eval } \\
(\mathbf{\%})\end{array}$ \\
\hline S1 & $\mu$ and $\beta$ & 2 & $\mathrm{C} 1, \mathrm{C} 3, \mathrm{Cz}, \mathrm{C} 2$ & 77.5 & 75 \\
\hline $\mathrm{S} 2$ & $\mu$ and $\beta$ & 2 & $\mathrm{C} 1, \mathrm{C} 3, \mathrm{Cz}, \mathrm{C} 2$ & 77.5 & 75 \\
\hline S3 & $\mu$ and $\beta$ & 2.25 & $\mathrm{C} 1, \mathrm{C} 3, \mathrm{C} 2, \mathrm{C} 4$ & 72.5 & 70 \\
\hline S4 & $\beta$ only & 5.5 & $\mathrm{C} 1, \mathrm{C} 3, \mathrm{Cz}, \mathrm{C} 2$ & 67.5 & 67.5 \\
\hline S5 & $\mu$ only & 6.25 & $\mathrm{C} 1, \mathrm{C} 3, \mathrm{Cz}$ & 66.25 & 72.5 \\
\hline S6 & $\mu$ and $\beta$ & 6.25 & $\mathrm{C} 1, \mathrm{C} 2, \mathrm{C} 4$ & 62.5 & 67.5 \\
\hline Mean & 70.62 & 71.25 \\
\hline
\end{tabular}

TABLE. 3 CLASSIFICATION ACCURACIES IN CASE OF MOTOR IMAGERY

\begin{tabular}{|c|c|c|c|c|c|}
\hline Sub & Band & $\begin{array}{c}\text { Time } \\
\text { (s) }\end{array}$ & Channel & $\begin{array}{c}\text { 5-CV } \\
(\%)\end{array}$ & $\begin{array}{l}\text { Eval } \\
(\%)\end{array}$ \\
\hline S1 & $\mu$ and $\beta$ & 1.25 & $\mathrm{C} 1, \mathrm{Cz}$ & 62.5 & 67.5 \\
\hline S2 & $\beta$ Only & 5.25 & $\mathrm{C} 1, \mathrm{C} 3, \mathrm{Cz}, \mathrm{C} 2$ & 62.5 & 72.5 \\
\hline S3 & $\mu$ and $\beta$ & 3.5 & $\mathrm{C} 1, \mathrm{Cz}, \mathrm{C} 2$ & 70 & 70 \\
\hline S4 & $\mu$ and $\beta$ & 6.5 & $\mathrm{Cz}, \mathrm{C} 2$ & 61.25 & 65 \\
\hline S5 & $\mu$ only & 4 & $\mathrm{C} 3, \mathrm{Cz}, \mathrm{C} 2, \mathrm{C} 4$ & 63.75 & 67.5 \\
\hline S6 & $\mu$ and $\beta$ & 1 & $\mathrm{C} 1, \mathrm{C} 3$ & 60 & 65 \\
\hline \multicolumn{4}{|c|}{ Mean } & 63.33 & 67.92 \\
\hline
\end{tabular}

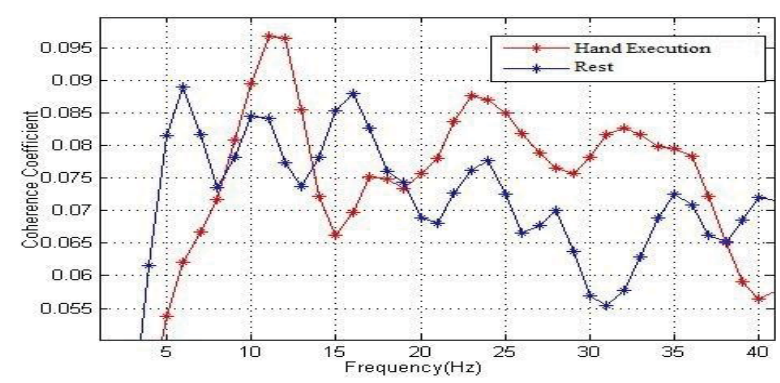

(a)

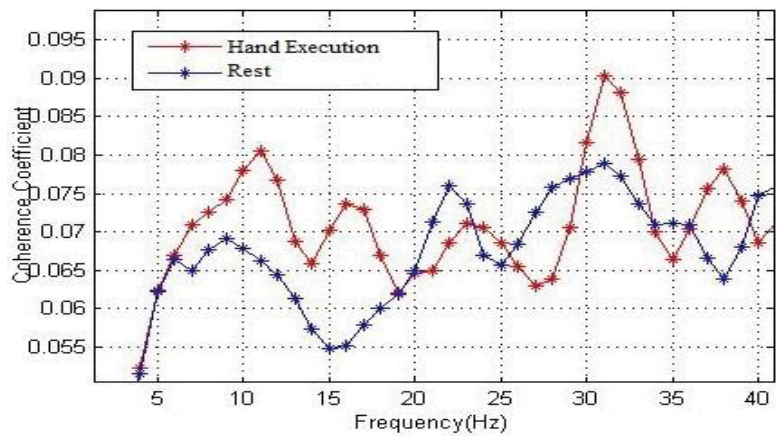

(b)

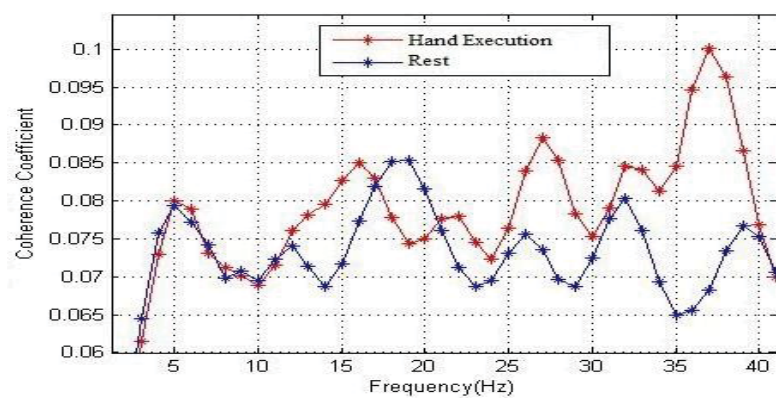

(c)

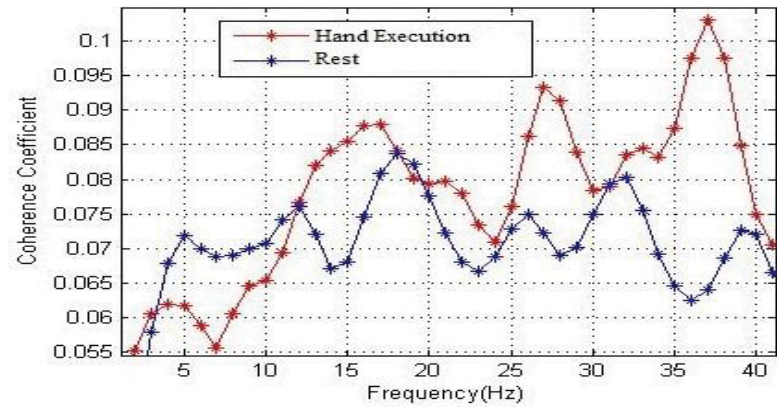

(d) 


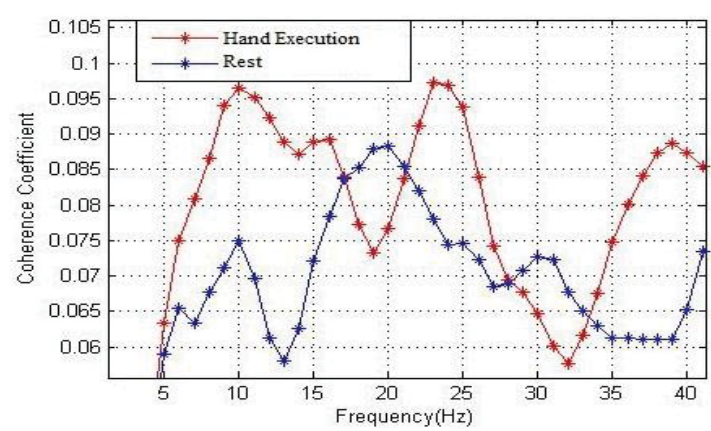

(e)

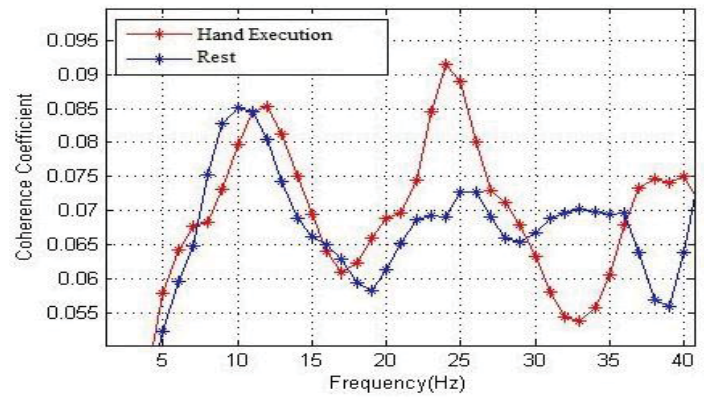

(f)

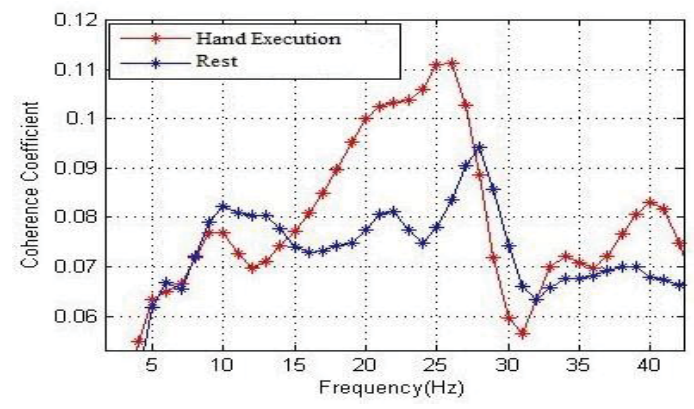

(g)

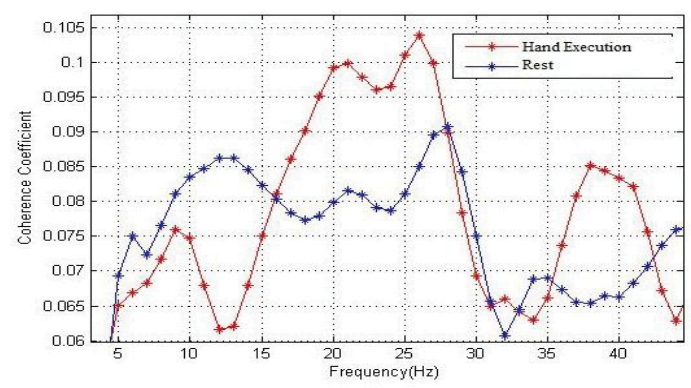

(h)

Fig.4. Coherence: between(a) CP3 and IF for S1, (b) CP4 and MF for S1, (c) CP3 and IF for S2, (d) CP4 and Thumb for S2, (e)CP3 and IF for S3, (f)CP3 and Thumb for S3, (g)CP3 and MF for S6, (h)CP4 and MF for S6.

In each table the combination of features types (bands), the time instant (s) of extracted features and the combination of EEG channels are also mentioned, which led us to the best possible accuracies. In all cases, 5-fold cross-validation (5$\mathrm{CV}$ ) and evaluation (Eval) accuracies are reported.
Among these novice subjects, four subjects have shown some significant cortico-muscular coupling in terms of coherence between the EEG and EMG signals of different channel combinations for the active finger motions only. For $\mathrm{S} 1$ the coupling of IF with EEG channels $\mathrm{C} 1, \mathrm{C} 2$ and $\mathrm{Cz}$ and the coupling of MF with $\mathrm{C} 4$ and $\mathrm{C} 2$ are found significant. For $\mathrm{S} 2$ the coupling of IF and TH with all the five EEG channels $\mathrm{C} 1, \mathrm{C} 3, \mathrm{Cz}, \mathrm{C} 4$ and $\mathrm{C} 2$ are found significant. S3 has also shown significant coupling of IF and TH with $\mathrm{C} 1, \mathrm{C} 3, \mathrm{Cz}$ and $\mathrm{C} 2$. Also the MF is found to be well coupled with $\mathrm{C} 1, \mathrm{C} 3$, $\mathrm{Cz}, \mathrm{C} 4$ and C2, in case of S6. Subjects S4 and S5 didn't show any distinguishable difference in coherence between hand execution and the rest state. Fig.4 shows some of these results, where the red line is showing the coherence pattern during the hand execution phase and the blue line is showing the coherence pattern in rest state. A paired t-test confirmed the difference between the coherence pattern in rest and hand movement conditions with the significance level $\mathrm{p}<0.01$ in all the cases. The experiments were conducted with full consent of the subjects as per the revised Helsinki Declaration of 2000 .

\section{DISCUSSION}

From the tables of classifier accuracies, it is observed that the average cross validation and evaluation accuracy, across all the subjects for active hand execution are $70.62 \%$ and $71.25 \%$ respectively. The same for passive movement using exoskeleton are $68.75 \%$ and $69.17 \%$ respectively and for motor imagery they are $63.33 \%$ and $67.92 \%$ respectively. From the results in Table. 1 to Table.3, it is obvious that the accuracy in active execution is better than passive execution which is in turn better than the motor imagery. These results also reflect the degree of involvement of the subjects during those three different tasks. However for stroke patients the ability to move fingers actively may be limited, and also if they are asked to perform the passive motion using exoskeleton then that may not be of much help in their recovery process due to lack of mental engagement. Therefore for an effective recovery it is important to drive the exoskeleton in assist-as-needed mode where the stroke sufferers will try to move their fingers actively but the lack of sufficient effort will be compensated by the exoskeleton assistance. To assess the effort generated by the subjects during the active finger movement, the coupling between the EEG signals and EMG signals generated by finger muscles will be quantified by looking at the coherence between them. From figures of coherence analysis (Fig.4) it is evident that the coherence coefficient during the hand execution is higher than the rest periods on an average and in particular frequency ranges it is significantly distinct from the rest. Specifically for S1, the coherence peak occurred at $11 \mathrm{~Hz}$ in case of IF and it is significantly higher than the rest in between $22 \mathrm{~Hz}$ to $35 \mathrm{~Hz}$, i.e. in higher beta and lower gamma bands. For the same subject the coherence peak for MF has occurred at $31 \mathrm{~Hz}$ and it is significantly distinct in $10-17 \mathrm{~Hz}$, i.e. mostly in alpha band and some lower beta band. S2 has shown peak coherence at $37 \mathrm{~Hz}$ both for IF and $\mathrm{TH}$, and the coherence is largely distinct from the rest, in between $30 \mathrm{~Hz}$ to $40 \mathrm{~Hz}$, i.e. the gamma band. In case of S3, the coherence peaks are found at $10 \mathrm{~Hz}$ and $23 \mathrm{~Hz}$ for IF, while it occurred at $24 \mathrm{~Hz}$ for $\mathrm{TH}$. The coherence in Hand Execution is significantly distinguishable from the rest between $15 \mathrm{~Hz}$ and 
$27 \mathrm{~Hz}$, with peak occurring at $25 \mathrm{~Hz}$, in case of MF of the $\mathrm{S} 6$. These results are found to be consistent for almost all the EEG channels that are used. The absolute value of the coherence is however much lower, as the muscle mass, driving the fingers are very small. Also the experiments were conducted for slow fingers motions with very small displacement without interacting with any obstruction. Therefore the muscle contraction was much less than the maximum limit, which could be the prime reason for obtaining such lower absolute values of the coherence coefficients. The important thing to notice is how much distinction we have got during finger motion relative to the no motion phase. This study is meaningful as it is targeted towards stroke sufferers who may have lost their finger mobility to a great extent. As these kinds of subtle finger movements require very less effort to conduct, therefore to distinguish them from the rest state is a challenge. These results show a lot of inter-subject variability both in case of training the classifiers and in coherence analysis. This study also shows the coupling of each individual finger with the brain, which could also give an indication of the engagement for the performance of each finger.

\section{CONCLUSION}

In the present investigation we have looked for an advanced approach of finger rehabilitation that takes into account the cortical activity related muscle activations which can be used to trigger a three finger hand exoskeleton for assisting people with hand disability to perform rehabilitation exercises. The system is also proposed to be supplemented by EEG analysis to cater for varying stages of disability (i.e., acute, and chronic) wherein the cortico-muscular coupling may be present in varying degrees. For this, we have checked the performance of an LDA classifier for three different modes of exercises; i.e., passive, active, and a kinesthetic imagination. The results of the EEG-EMG coherence analysis and EEG analysis alone have shown high potential for effectiveness of the proposed device, as we are in a position to predict with a good degree of certainty, when the persons is actually making an effort to move their fingers. But there are still some challenges to deal with, such as the intersubject variability and dynamic shift in frequency due to various neurological reasons and fatigue. Therefore the system needs to be upgraded further to adapt to these changes. Also we need to make the system ready for on-line testing on stroke affected people with loss of finger mobility, and the exoskeleton should also be equipped with sensors for assist-as-needed mode control.

\section{ACKNOWLEDGEMENT}

This work is supported by Department of Science and Technology (DST), India and UK India Education and Research Initiative (UKIERI).

\section{REFERENCES}

[1] World Health Organization: Global Report: 2002 http://www.who.int/whr/2002/en/index.html.

[2] Page SJ, Levine P, Leonard AC: Mental practice in chronic stroke: Results of a Randomized, Placebo-controlled trial, Stroke 2007, 38(4):1293-1297.

[3] Farmer J, Zhao X, van Praag H, Wodtke K, Gage FH, Christie BR, Effects of voluntary exercise on synaptic plasticity and gene expression in the dentate gyrus of adult male Sprague-Dawley rats in vivo, Neuroscience, vol.124, pp.71-79, 2004

[4] N.S.K. Ho, K.Y. Tong, An EMG-driven Exoskeleton Hand Robotic Training Device on Chronic Stroke Subjects, IEEE International Conference on Rehabilitation Robotics, Rehab Week Zurich, ETH Zurich Science City, Switzerland, June 29 - July 1, 2011.

[5] Andrade and Andrade, On The Relationship between Features Extracted from EMG and Force for Constant and Dynamic Protocols, 34th Annual International Conference of the IEEE EMBS San Diego, California USA, 28 August 1 September, 2012.

[6] B.B. Bhakta, "Management of spasticity in stroke," British Medical Bulletin, vol. 56, pp. 476-485, 2000.

[7] G. Prasad, P. Herman, D. Coyle, S. McDonough, J.Crosbie,Applying a brain-computer interface to support motor imagery practice in people with stroke for upper limb recovery: A feasibility study, Journal of NeuroEngineering and Rehabilitation, 7:60, 2010.

[8] F. Cincotti,F. Pichiorri, P. Arico, F. Aloise, F. Leotta1, F. de VicoFallani, J. del R. Millan, M. Molinari, and D. Mattia,EEG-based Brain-Computer Interface to support post-stroke motor rehabilitation of the upper limb, 34th Annual International Conference of the IEEE EMBS, San Diego, California USA, 28 August - 1 September, 2012.

[9] Mima T, Hallett M (1999) Corticomuscular coherence: a review. J Clin Neurophysiol 16: 501-511.

[10] Pfurtscheller G, Müller GR, Pfurtscheller J, Gerner HJ, Rupp R (2003) 'Thought'--control of functional electrical stimulation to restore hand grasp in a patient with tetraplegia. Neurosci Lett 351: 33-36.

[11] Baker MR, Baker SN (2003) The effect of diazepam on motor cortical oscillations and corticomuscular coherence studied in man. J Physiol 546: 931-942.

[12] Armstrong DM (1988). The supraspinal control of mammalian locomotion. J Physiol 405, 1-37..

[13] Y. Hashimoto, J. Ushiba, A. Kimura, M. Liu and Y. Tomita, Correlation between EEG-EMG coherence during isometric contraction and its imaginary execution, ACTA Neurobiol Exp 2010, 70: $76-85$

[14] F. Meng, K.Y. Tong, S. T. Chan, W. W. Wong, K. H. Lui, K.W. Tang,X. R. Gao, "Study on connectivity between coherent central rhythm and electromyographic activity", Journal of Neural Engineering, vol.5, pp.324-332, 2008.

[15] F. Meng, K.Y. Tong, S. T. Chan, W. W. Wong, K. H. Lui, K.W. Tang,X. R. Gao, "Cerebral plasticity after subcortical stroke as revealed by cortico-muscular coherence", IEEE Transaction on Neural Systems and Engineering, vol. 17, pp.234-243, 2009.

[16] T. Shibata, Y. Suhara, T. Oga, Y. Ueki, T. Mima, Shin Ishii, Application of multivariate autoregressive modeling for analysising interaction between EEG and EMG in humans, International Congress Series $1270(2004)$ pp.249-253.

[17] D. Tuncel, A. Dizibuyuk, M. K. Kiymik, Time Frequency Based Coherence Analysis Between EEG and EMG Activities in Fatigue Duration, Journal of Medical Systems, April 2010, Volume 34, Issue 2, pp 131-138, Date: 28 Oct 2008

[18] Pfurtscheller G, Graimann B, Huggins JE, Levine SP, Schuh LA, Spatiotemporal patterns of beta desynchronization and gamma synchronization in corticographic data during self-paced movement, Clin. Neurophysiol. 2003; 114:1226-1236

[19] T. H. Petersen, M.Willerslev-Olsen, B. A. Conway and J. B. Nielsen, The motor cortex drives the muscles during walking inhuman subjects, J Physiol 590.10 (2012) pp 2443-2452.

[20] Shyam Sunder Nishad, Ashish Dutta, Anupam Saxena. "Design and Control of a Three Finger Hand Exoskeleton for Translation of a slender object". Proceedings of the International Conference on Ubiquitous Robots and Ambient Intelligence, Kuala Lumpur, Malaysia 2014, pp. 179-184. 